\title{
Co-variation in time between near-far accommodation of the lens and trapezius muscle activity
}

\author{
Mikael Forsman ${ }^{\mathrm{a}, \mathrm{b}, *}$, Camilla Lodin ${ }^{\mathrm{b}}$, and Hans Richter ${ }^{\mathrm{b}}$ \\ ${ }^{a}$ Division of Occupational and Environmental Medicine, Department of Public Health Sciences, Karolinska \\ Institutet, SE-171 76 Solna, Sweden. \\ ${ }^{\mathrm{b}}$ Center for Musculoskeletal Research, Department of Occupational and Public Health Sciences, Faculty of \\ Health and Occupational Studies, University of Gävle, SE-801 76 Gävle, Sweden.
}

\begin{abstract}
Visual strain and discomfort may contribute to the generation of work-related musculoskeletal disorders among e.g. computer workers. A positive correlation on a group level between eye-lens accommodation and trapezius muscle activity has been reported. In this study we investigated the possibility of a direct, fast, connection between lens accommodation and trapezius muscles activity. The subjects focused alternately on Near and Far targets, with a mean switch time of $5 \mathrm{~s}$, through four different lenses. The cross-correlation, $\mathrm{R}(\mathrm{tau})$ was computed, between the time signals of accommodation and electromyography (EMG) from 23 subjects. In the overall mean R(tau) of 736 curves, a small but significant correlation peak (0.019) with a delay (of the EMG signal) of about $0.3 \mathrm{~s}$, revealed a small common component in the two signals. Among the lenses, the positive lens (3.5 D), showed the highest correlation peak (0.040). The correlation may be caused by a direct "hard-wired" connection between the ciliary and trapezius muscles. But it could also be caused indirectly by the subject's need for a more stable head in a more demanding visual task. The latter is supported by the result that the correlation was the highest in the positive lens condition. The present correlation is however weak and it has probably a low practical importance.
\end{abstract}

Keywords: Visual discomfort, cross-correlation, electromyography, visual strain, ciliary muscle

\section{Introduction}

Work-related musculoskeletal disorders (MSDs) in the neck/shoulder are common problem among e.g. computer workers. In a cross-sectional study of employees at 28 different call-centers [1], a significant positive association was found between eyestrain and neck-shoulder symptoms; $46 \%$ of the 1162 questionnaire responders reported neck-shoulder symptoms, about half of these also reported eyestrain. Helland and co-workers investigated lightning and neck/ shoulder disorders among 34 computer worker before and after moving to an office landscape. The office landscape had large windows placed behind the visual display units (VDUs). Visual discomfort increased after moving to the office landscape, and it explained
$53 \%$ of the variance in the neck and shoulder pain [2].

As indicated by the studies above, visual discomfort may constitute one path, contributing to the generation of MSDs. A neurological connection has also been supported in laboratory studies, see e.g. [3]. The Cinderella hypothesis [4] suggests that low-threshold motor-units are at risk for selective overuse, and that a small increase of the "static" muscular activity may increase the risk of motor-unit overuse and the risk for MSDs.

The experiment of the present study had two parts. In part one [5-6],twenty-eight subjects focused a target at Near $(22.2 \mathrm{~cm})$ or Far $(3.0 \mathrm{~m})$ in five-minuteperiods through one of four different optical trial lenses $(-3.5 \mathrm{D},+3.5 \mathrm{D}, 0 \mathrm{D}$ and prism lenses $1-2 \mathrm{D})$. In the $-3.5 \mathrm{D}$ near condition, subjects who had a high

${ }^{*}$ Corresponding author. E-mail: mikael.forsman@ki.se 
accommodative response also showed a higher static trapezius muscle activity level [5]. Hence, there was a group correlation between mean accommodation load at a near target and trapezius muscular activity. Since the periods of static ocular load were $5 \mathrm{mi}-$ nutes, it was not possible to conclude if this was a direct effect (within a second), that actually depended on the ciliary load, or if it was a slower indirect effect.

The purpose of this study was to investigate the possibility of a direct, fast, connection between the ciliary and trapezius muscles, by studying the crosscorrelation between the time signals of ciliary muscle activity (obtained indirectly from autorefractor measurements) and trapezius muscle activity.

\section{Methods}

This study was carried out as a part of a larger data collection. For details on the subjects, visual conditions, and measurements, see [6].

\subsection{Subjects}

Twenty-eight subjects (18 females and 10 males), with a mean age of 29 (range 19-42, std 8) were included in the study. Fifteen of the subjects (mean age 27 , range $19-42$, std 8 ) were healthy and thirteen had a history of eye disorder (asthenopia) and nonspecific neck disorders (mean age 32, range 21-42, std 7). The patient group had been referred by general doctors or optometrists to an orthoptist for further investigation and treatment. Any prescribed orthoptic treatment had been completed 2-3 months prior to their participation in the present study. Informed consent was given by each subject before participation. The study was approved by the Uppsala University Medical Ethical Review Board.

\subsection{Procedure}

During the experiment the subjects were seated in an office-chair in a slightly leaning back posture, supported by a combined head and backrest adjusted to their morphology. The subject's upper arms were alongside the trunk. Their hands were stationary and resting, either on their lap or on adjusted armrests. Because the eyes had to be aligned to the axis of the optometer, movements from the neck/scapular area were not allowed. The high contrast targets consisted of a bright $\mathrm{X}$ illuminated from behind with a polyc- hromatic LED (Light Emitting Diode, LED) (Everlight Electronics Co., Ltd., England) which emitted a white color. One target (Near) was placed $5 \mathrm{D}$ away from the age-appropriate near point of accommodation and the other target (Far) was placed $3.0 \mathrm{~m}$ away.

The subjects were asked to alternately focus at the Near and the Far target. In each trial, the subjects focused at Near 15 times and at Far 15 times. Mean change time between Near and Far was 5 s (3-7s). Varying magnitudes of defocus blur were introduced into the optical axis of the viewing eyes via one of four optical trial lenses: 0 diopter (D) lenses; -3.5 D lenses; individually adjusted prism D lenses (1-2 D base out); and+3.5 D lenses. The subject performed two Near-Far trials times per lens.

\subsection{Optometric recordings}

The accommodative/vergence response was assessed using an infrared autorefractor (PowerRefractor R 03, Plusoptix, Nurnberg, Germany) based on the principles of photorefraction. The photorefraction technique analyzes the vergence of rays that are returned from the eye after reflecting an illuminated spot on the retina. The accommodative responses were expressed in dioptres (D).

\subsection{Electromyography and electrocardiography}

Electromyography (EMG) was collected bilaterally from the descending part of the upper trapezius muscle. The skin was cleansed with alcohol and rubbed with a fine cloth. Two disposable $\mathrm{Ag}-\mathrm{AgCl}$ electrodes were placed along the direction of the muscle fibers with a center-to-center distance of 2 $\mathrm{cm}$. The electrodes were centered $2 \mathrm{~cm}$ lateral of the mid-point of the line connecting vertebra $\mathrm{C} 7$ and acromion [7].

Electrocardiography (ECG) was recorded and used to filter out heart signal disturbances in the EMG signals.

The EMG and ECG signals were amplified, highpass filtered $(30 \mathrm{~Hz})$, and sampled at $2000 \mathrm{~Hz}$ (EMG100C, BIOPAC Systems, Inc., Santa Barbara, CA, USA). The EMG data were normalized to the root-mean-square (RMS) value of the middle $10 \mathrm{~s}$ of 15-s submaximal contractions (the reference voluntary electrical activity; RVE). During the reference contractions both arms were straight and horizontal in abduction, which corresponds to $15-20 \%$ of a maximal voluntary contraction [7]. The mean value of 
three repeated RVE contractions was used to normalize and express the measurement data in \%RVE. The EMG recordings were RMS-converted in 100-ms windows and quadratically adjusted for noise (the lowest 400-ms moving RMS value of a recording during relaxation).

The raw EMG signals were often, especially on the left side, disturbed by additive heart signals. This disturbance was rejected by estimation of the heart signal additions via the estimated timings of the Rpeaks, and by subtracting these additions from the EMG signals.

\subsection{Data analyses}

The co-variation in time between the refraction signal of the accommodation of the lens and the RMS transformed trapezius muscular activity was analyzed by the use of cross-correlation functions, R(tau). This function computes the correlation coefficient between two signals, at different time delays (tau) of one of the signals. When tau is zero and the function is normalized, $\mathrm{R}(\mathrm{tau}=0)$ equals the Pearson's correlation coefficient. If the two signals are identical, $\mathrm{R}(\mathrm{tau}=0)$ is equal to 1 . If there is a co-variation in time between the two signals, i.e. one signal effects the other or vice versa, R(tau) will be significantly different from zero, and the tau for which the maximum amplitude occurs in $\mathrm{R}(\mathrm{tau})$, is an estimate of the effect delay.
The sampled EMG signals were filtered 20-500 $\mathrm{Hz}$, RMS-transformed (200 ms), and interpolated to the sampling frequency of the autorefractor signal, 25 $\mathrm{Hz}$. Both signals were then filtered $0.05-5 \mathrm{~Hz}$. Normalized cross-correlation functions, R(tau), were computed and saved for the 32 combinations (two Near-Far trials, four lens-conditions, left and right eye, and left and right trapezius muscles). A mean curve was calculated from all $736 \mathrm{R}$ (tau)-curves.

Two mean R(tau)-curves, each from 92 traces, were also calculated within each lens-condition, one for left trapezius muscle and one for right trapezius muscle.

\section{Results}

Signals of adequate quality were obtained from 23 of the subjects, which were included in the analyses of cross-correlation.

In the overall mean $\mathrm{R}(\mathrm{tau})$ from 736 traces, a small but significant correlation peak, $\mathrm{R}(\mathrm{tau})=0.019$, was seen at tau about $0.3 \mathrm{~s}$. That means that the correlation between the EMG-signal and the refraction signal was highest in the EMG-signal approximately 0.3 $\mathrm{s}$ after the refraction signal. $\mathrm{R}(\mathrm{tau})$ also showed a wave pattern with hills of descending amplitude at taus around the multiples of $10 \mathrm{~s}$, on both sides of zero (see Figure 1). These hills agree in time with the trials mean time of the alternating Near-Far focusing.

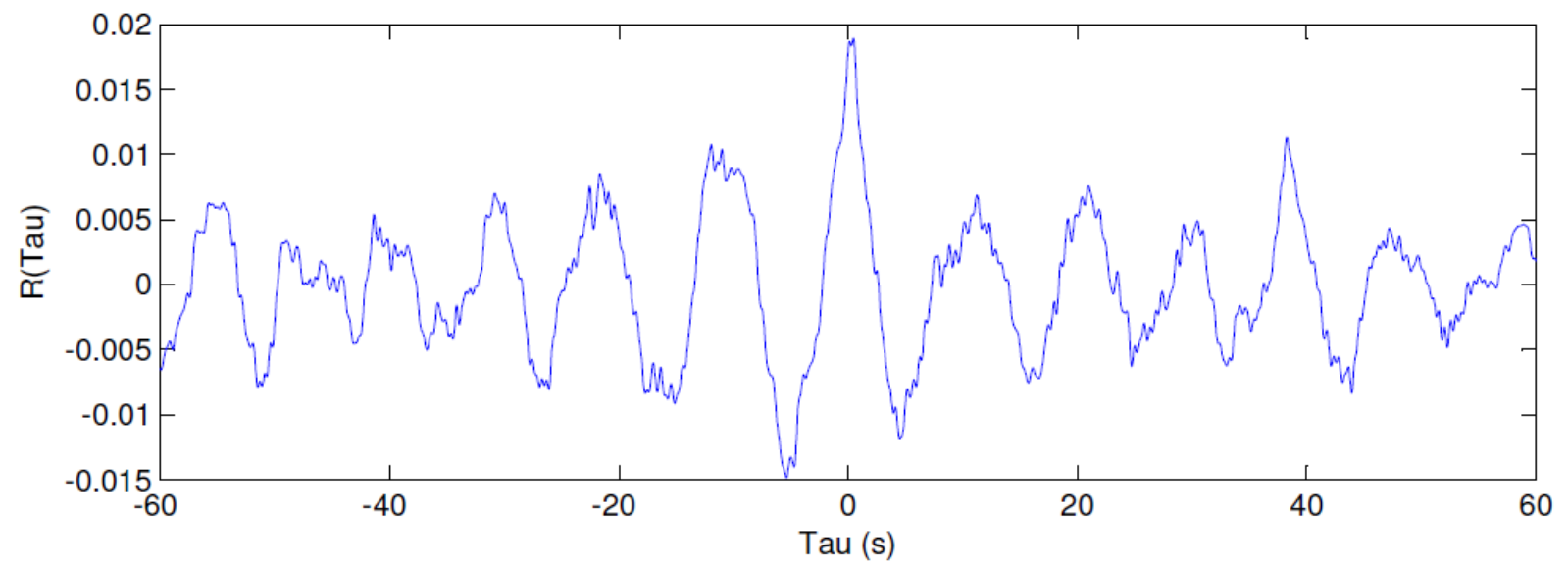

Fig. 1. Cross-correlation function between EMG RMS and refraction signals. Mean of 23 subjects' two Near-Far trials, four lensconditions, left and right eye, and left and right trapezius muscles, i.e., mean from $736 \mathrm{R}(\mathrm{tau})$-curves. The top peak at tau $\approx 0.3 \mathrm{~s}$ means that the two signals are related and that the very small component of the refraction signal in the EMG RMS is delayed, and lies approximately 0.3 after the refraction signal. 
The eight lens specific $\mathrm{R}(\mathrm{tau})$-curves showed a similar wave pattern. The most clear and evident pattern of eye-trapezius muscle correlation was seen for the positive lens, for both muscles. Mean R(tau)curves for lens condition +3.5 and -3.5 (left trapezius) are shown in figure 2.
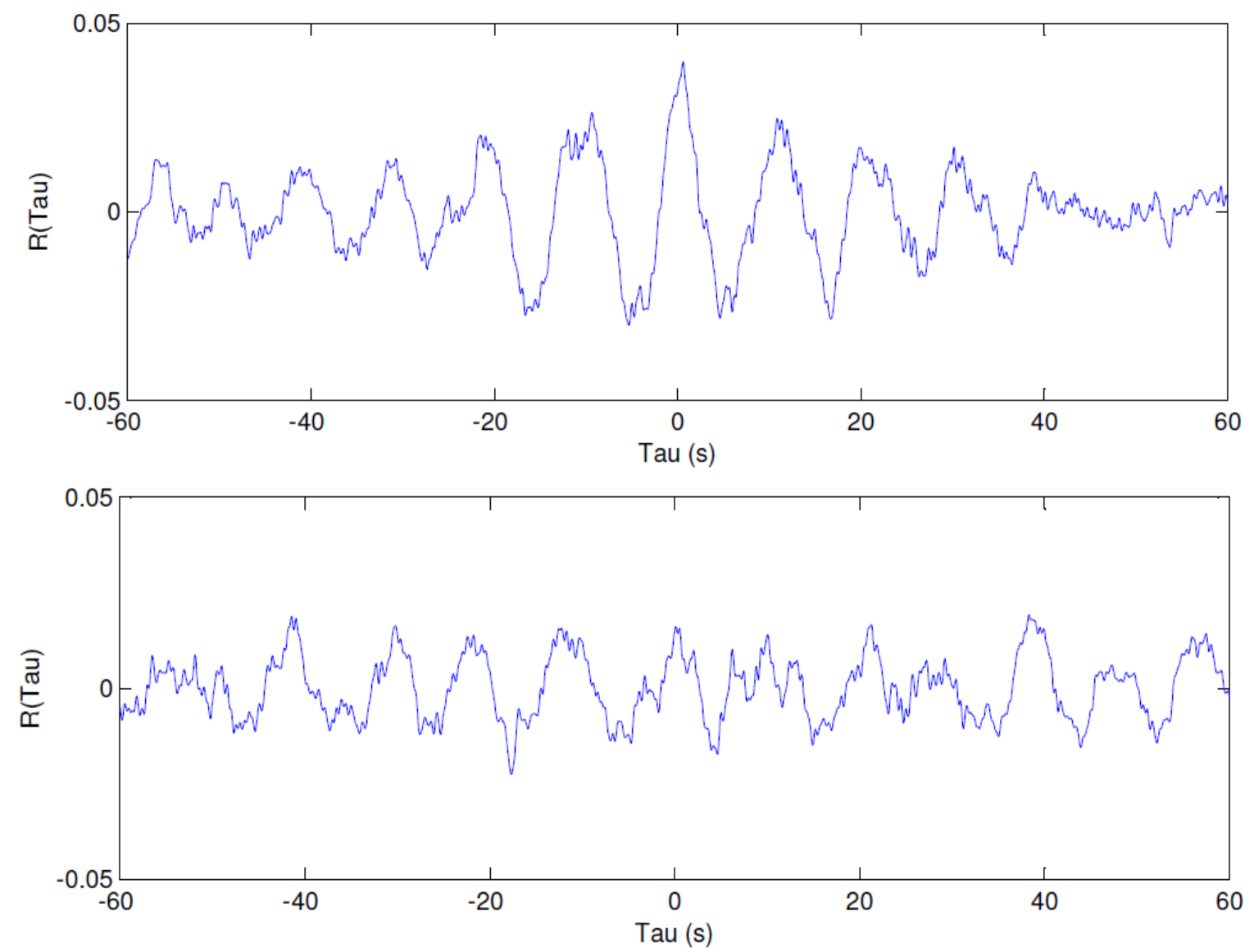

Fig. 2.Cross-correlation function between EMG RMS and refraction signals, mean of 23 subjects, two Near/Far trails, and left and right eyes, hence, mean of $92 \mathrm{R}(\mathrm{tau})$ traces. Both diagrams shows the mean R(tau) for left trapezius vs. refraction signal. The top diagram shows the condition of most evident cross-correlation, with the positive lens $(+3.5 \mathrm{D})$. The lower diagram shows the minus lens condition $(-3.5 \mathrm{D})$.

\section{Discussion}

The results show a small co- variation in time between the signal of eye lens Near-Far accommodation and the trapezius muscle activity pattern. Although the mean R(tau) peak value was small, because the large total number of Near, Far, Near switches, and since both the muscle and refraction signals are high pass filtered, the overall mean of $\mathrm{R}(0.3)$ where the maximal peak was found was estimated to be significant with a p-value below 0.001 .
Both signals include random components, mostly biological variance for the EMG, and technical noise for the refraction signal. These components are not expected to correlate with each other. A harder filtration with a low-pass filter of lower cut-off frequency would increase the R(tau) peak values. Mean R(tau) was the highest for the positive lens, but differences between the lenses are not yet statistically tested.

The significant positive correlation in the mean curves means that shortly after the accommodation signal is increasing, the muscular activity of the trapezius muscle also increases to some extent, (by the mean). This correlation is theoretically interesting. It 
may be caused by a direct "hard-wired" connection between the ciliary and trapezius muscles, since there is a low time delay between the signals.

The precision of the delay time of the mean $\mathrm{R}(\mathrm{tau})$, $0.3 \mathrm{~s}$, is low because of the unsharp peak, it may be longer. A longer delay time was also indicated in the case of the positive lens, which had the highest lens specific R(tau), with a sharper peak. So the correlation could also be caused indirectly by the subject's need for a more stable head in a visually demanding near viewing task. The results illustrated in Figure 2 may rather support the latter interpretation. The minus lens induces the highest accommodation load, and highest ciliary muscle activity, but the correlation amplitude was the highest in the positive lens condition.

The present correlation is however weak; it could not be revealed without extensive statistical analyses. Therefore, the practical importance is probably low. The correlation found on group level [5] is higher than the present mean correlations at the individual level. This indicates that there also may be an indirect link from visual demands to increased muscular activity response after longer time than the $5 \mathrm{~s}$ between the Near-Far alterations used here.

\section{References}

[1] C. Wiholm, H. Richter, S.E. Mathiassen and A. Toomingas, A, Associations between eyestrain and neck-shoulder symptoms among callcenter operators, Scandinavian Journal of Work, Environment \& Health, Suppl. 3 (2007), 54-59.

[2] M. Helland, G. Horgen, T.M. Kvikstad, T. Garthus, J.R. Bruenech and A. Aaras, Musculoskeletal, visual and psychosocial stress in VDU operators after moving to an ergonomically designed office landscape, Applied Ergonomics 39 (2008), 28495.

[3] H.O. Richter, J. Andersson, H. Schneider and B. Långström, Neuroanatomical correlates of voluntary inhibition of accommodation/vergence under monocular open-loop viewing conditions, European Journal of Neuroscience 21 (2005) 30773088.

[4] G.M. Hägg, Static work and myalgia - a new explanation model, in:PA Andersson, DJ Hobart, JV Danoff eds., Electromyography and kinesiology, Elsevier Science, Amsterdam (1991), pp. 115-199.

[5] O. Richter, T. Bänziger and M. Forsman, Eye-lens accommodation load and static trapezius muscle activity, European Journal of Applied Physiology, 111 (2011), 29-36.Vision research?

[6] O. Richter, T. Bänziger, S Abdi and M. Forsman, Stabilization of gaze: a relationship between ciliary muscle contraction and trapezius muscle activity, Vision Research 50 (2010), 255969.

[7] S.E. Mathiassen, J. Winkel and G.M. Hägg, Normalization of surface EMG amplitude from upper trapezius muscle in ergonomic studies - A review, Journal of Electromyography and Kinesiology 5 (1995), 197-226. 